\title{
Diagnosis of acute respiratory distress syndrome (DARTS) by bedside exhaled breath octane measurements in invasively ventilated patients: protocol of a multicentre observational cohort study
}

\author{
Laura A. Hagens ${ }^{1}$, Nanon F. L. Heijnen ${ }^{2}$, Marry R. Smit ${ }^{1}$, Alwin R. M. Verschueren ${ }^{3}$, \\ Tamara M. E. Nijsen ${ }^{3}$, Inge Geven ${ }^{3}$, Marcus J. Schultz ${ }^{1,4,5}$, Dennis C. J. J. Bergmans ${ }^{2}$, \\ Ronny M. Schnabel ${ }^{2}$, Lieuwe D. J. Bos ${ }^{1,6}$; on behalf of the DARTS consortium*
}

${ }^{1}$ Department of Intensive Care, Amsterdam UMC, Location AMC, University of Amsterdam, Amsterdam, The Netherlands; ${ }^{2}$ Department of Intensive Care, Maastricht University Medical Center+, Maastricht, The Netherlands; ${ }^{3}$ Sleep \& Respiratory Solutions, Philips Research, High Tech Campus, Eindhoven, The Netherlands; ${ }^{4}$ Mahidol-Oxford Tropical Medicine Research Unit (MORU), Mahidol University, Bangkok, Thailand; ${ }^{5}$ Nuffield Department of Medicine, University of Oxford, Oxford, UK; ${ }^{6}$ Department of Respiratory Medicine, Amsterdam UMC, Location AMC, University of Amsterdam, Amsterdam, The Netherlands

Contributions: (I) Conception and design: All authors; (II) Administrative support: None; (III) Provision of study materials or patients: None; (IV) Collection and assembly of data: None; (V) Data analysis and interpretation: None; (VI) Manuscript writing: All authors; (VII) Final approval of manuscript: All authors.

Correspondence to: Laura A. Hagens. Department of Intensive Care, Amsterdam UMC, Location AMC, University of Amsterdam, Meibergdreef 9, Amsterdam, 1105 AZ, The Netherlands. Email: 1.a.hagens@amsterdamumc.nl.

\begin{abstract}
Background: Acute respiratory distress syndrome (ARDS) is currently diagnosed by the Berlin Definition. Diagnosis is subjective and often late. Untargeted metabolomics analysis of exhaled breath with gas chromatography and mass spectrometry (GC-MS) showed that the breath concentration of octane has a high diagnostic accuracy for ARDS. To facilitate rapid bedside measurement of this biomarker, a point-ofcare (POC) breath test was developed. A prototype already showed good reproducibility and repeatability for the detection of octane. In this study we aim to measure octane in exhaled breath of invasively ventilated intensive care unit (ICU) patients and validate the diagnostic accuracy of the breath test for the early diagnosis of ARDS.

Methods: This is a multicentre observational cohort study in patients admitted to the ICU receiving invasive ventilation for at least 24 hours. At least 500 patients in two academic hospitals in The Netherlands will be included. ARDS patients will be compared to patients without ARDS. ARDS diagnosis will be based on the Berlin Definition. Two diagnostic assessments will be performed during the first 72 hours of invasive ventilation, including breath sampling, arterial blood gas analysis and lung ultrasound (LUS). In patients fulfilling the criteria for ARDS, three additional breath samples will be taken to assess resolution. The primary endpoint is the diagnostic accuracy for ARDS, defined by the area under the receiver operating characteristics curve (AUROCC) of octane concentration in exhaled breath. Secondary endpoints are the association between exhaled breath octane and ARDS adjusted for confounders, and the added diagnostic accuracy of the breath test on top of the Lung Injury Prediction Score (LIPS).
\end{abstract}

\footnotetext{
*, members of the DARTS consortium: Amsterdam UMC: Laura A. Hagens, Marry R. Smit, Marcus J. Schultz, Lieuwe D. J. Bos; Maastricht University Medical Center: Nanon F. L. Heijnen, Dennis C. J. J. Bergmans, Ronny M. Schnabel; Philips Research: Alwin R. M. Verschueren, Tamara M. E. Nijsen, Inge Geven.
} 
Discussion: This is the first study that validates a metabolic biomarker of ARDS in an adequate sample size. The major novelty is the use of a POC breath test that has been specifically developed for the purpose of diagnosing ARDS. Strengths are; assessment in the early phase, in patients at risk for ARDS, longitudinal sampling and an expert panel to reliably diagnose ARDS. This study will provide a decisive answer on the question if exhaled breath metabolomics can be used to diagnose ARDS.

Trial registration: The trial is registered at trialregister.nl (ID: NL8226) with the tag "DARTS".

Keywords: Acute respiratory distress syndrome (ARDS); diagnosis; exhaled breath; biomarker; metabolomics; intensive care

Submitted Mar 22, 2021. Accepted for publication May 28, 2021.

doi: $10.21037 / \mathrm{atm}-21-1384$

View this article at: https://dx.doi.org/10.21037/atm-21-1384

\section{Introduction}

The acute respiratory distress syndrome (ARDS) is a severe complication of critical illness characterized by protein rich pulmonary oedema (1). ARDS affects approximately 10\% of patients admitted to the intensive care unit (ICU) (2) and is associated with a mortality rate of $40 \%$. ARDS is currently diagnosed according to the criteria formulated in the Berlin Definition (3). ARDS is defined as the acute development of hypoxemia requiring $5 \mathrm{cmH}_{2} \mathrm{O}$ of positive end-expiratory pressure or more with bilateral infiltrates on chest radiography indicative of pulmonary oedema within 7 days of a known clinical risk factor that is not completely explained by a cardiogenic cause (3). However, the interpretation of chest radiography remains subjective and is associated with low interrater agreement (4). In general, macroscopic oedema visible on chest radiography is regarded a late sign of lung injury caused by increased vascular permeability. Furthermore, diagnosis is frequently missed, leading to inadequate treatment and ventilation strategies in these patients $(2,5)$. Current tests are neither sensitive nor specific (6). Therefore, two of the greatest challenges in ARDS are early and objective recognition of ARDS.

Exhaled breath contains hundreds of volatile organic compounds (VOCs) that are reflective of metabolic changes in the lungs (7). Gas chromatography and mass spectrometry (GC-MS) is considered the gold standard for VOC detection. This technique was previously used to discover and validate a three-metabolite diagnostic model with good accuracy for ARDS (8). The concentration of octane in exhaled breath explained most of the diagnostic accuracy and octane is linked to ARDS pathophysiology in clinical and preclinical studies (9). ARDS causes an increase in oleic acid in the circulation. At the same time, oxidative stress causes an increase in release of reactive oxygen species which leads to increased lipid peroxidation. Lipid peroxidation of oleic acid generates octane, making this a possible explanation for increased levels of octane in ARDS $(10,11)$.

Exhaled breath analysis by GC-MS is not suitable for diagnosing ARDS in clinical practice. To maintain reproducible results, GC-MS is labour intensive and requires specialized personnel. It operates in vacuum and requires helium as a carrier gas for optimal results. These factors limit the use of GC-MS for breath analysis in a clinical and time-sensitive environment such as the ICU. To overcome the disadvantages of GC-MS, a point-of-care (POC) breath test has been developed by Philips Research (Philips Research, Global Headquarters, Eindhoven, The Netherlands). It is smaller in size than the GC-MS, does not require a vacuum and does not need helium as carrier gas, but operates on medical air. Therefore, it could enable bed-side measurements and opens the possibility of monitoring in the near future. The working prototype showed good reproducibility and repeatability for octane detection in parts per billion (ppb) (12).

Taken together, there is a clear need for a POC breath test that can selectively quantify octane concentrations in exhaled breath and can provide a result within hours, without the need of highly specialized personnel. We hypothesize that the octane concentration in exhaled breath, as measured with a POC breath test, can facilitate early diagnosis of ARDS in invasively ventilated ICU patients. We will assess the optimal threshold and report the diagnostic accuracy, as reflected by characteristics like the sensitivity and specificity. We will compare and combine the diagnostic accuracy of the breath test with other noninvasive methods that can be used to quantify ARDS risk, 
such as the Lung Injury Prediction Score (LIPS) (13), lung ultrasound (LUS) (14) and plasma biomarkers (15-17).

\section{Methods}

\section{Design}

This is a multicentre observational cohort study with longitudinal sampling of consecutive patients admitted to the ICU receiving invasive ventilation for at least 24 hours. The study will be performed in two academic hospitals in The Netherlands (Amsterdam UMC, Location AMC and Maastricht University Medical Center). Patients will be recruited in a 2-year time period. The Institutional Review Board of the Academic Medical Center, Amsterdam, The Netherlands, and of the Maastricht University Medical Center+, Maastricht, The Netherlands, waived the need for ethical approval of the protocol (W18_311 \#18.358 and 2019-1137). The biobank review board of the Academic Medical Center Biobank approved the development of a biobank for the study (approval 2018_287\#A201921). The study is funded by Health Holland via the Dutch Lung Foundation. The trial is registered at trialregister.nl (ID: NL8226) with the tag "DARTS study".

\section{Informed consent}

Patients will be recruited as soon as possible after start of mechanical ventilation by a team of researchers. Therefore, all included patients are, per definition, incapacitated to provide informed consent. All measurements in this study are non-invasive and data needs to be collected shortly after intubation. For this reason, written informed consent cannot be obtained from the patient beforehand and deferred consent will be asked as the alternative. Patients will be informed and asked for consent shortly after regaining the capacity to provide consent. If the patient declines participation in the study, the collected data and all biological samples will be destroyed.

If the patient deceases, the General Data Protection Regulation (GDPR) states that the obtained data can be used without consent. If a patient does not regain capacity before discharge, the representative will be asked for deferred consent. In case the representative does not give consent, the collected data will be destroyed. When the representative could not be reached, and the patient does not regain capacity, the patient will be excluded and the collected data and all biological samples will be destroyed.
In case the representative gave consent, the patient will have the possibility of an opt-out procedure. This procedure is based on the exception criteria of the GDPR where processing without explicit informed consent is necessary for the purposes of the legitimate interests pursued, in this case the scientific soundness of the results. If consent is not given by either the patient or the representative, data and all biological samples will be destroyed.

\section{Study population}

The DARTS study will enrol patients admitted to the ICUs of the two participating hospitals who are expected to receive invasive ventilation for at least 24 hours and are 18 years or older. Patients who received invasive ventilation for more than 48 hours at any moment in the 7 days preceding the moment of inclusion or who have a life expectancy of less than 24 hours at the moment of inclusion, will be excluded. Tracheotomised patients will also be excluded. Finally, patients will be excluded if breath sampling is deemed clinically inappropriate or if consent is withdrawn by the patient or family.

\section{Study procedures}

Patients will be recruited within the first 48 hours after the start of invasive ventilation. Baseline clinical data is recorded, including: patient characteristics, comorbidities, measures of disease severity such as Acute Physiology and Chronic Health Evaluation (APACHE) II (18), Sequential Organ Failure Assessment (SOFA) (19) and risk factors for ARDS. During the first 48 hours of invasive mechanical ventilation, the first assessment will be performed, followed by a second measurement a day later. Both assessments include: breath sampling, arterial blood gas analysis and a LUS exam (Figure 1). Additionally, the following parameters will be collected; ventilation and oxygenation parameters, current medication and antibiotics. The chest radiography and worst $\mathrm{PaO}_{2} / \mathrm{FiO}_{2}$ ratio in the 24 hours before breath sampling will be collected and used for ARDS diagnosis according to the Berlin criteria.

If the patient fulfils the Berlin criteria for ARDS during the first 2 days of invasive mechanical ventilation according to the researcher, three additional breath samples will be taken, at approximately 48 hours, 96 hours and 12 days after inclusion. These three additional measurements are used to monitor the disease progress of the patients with ARDS. During the third assessment LUS will be repeated while the 


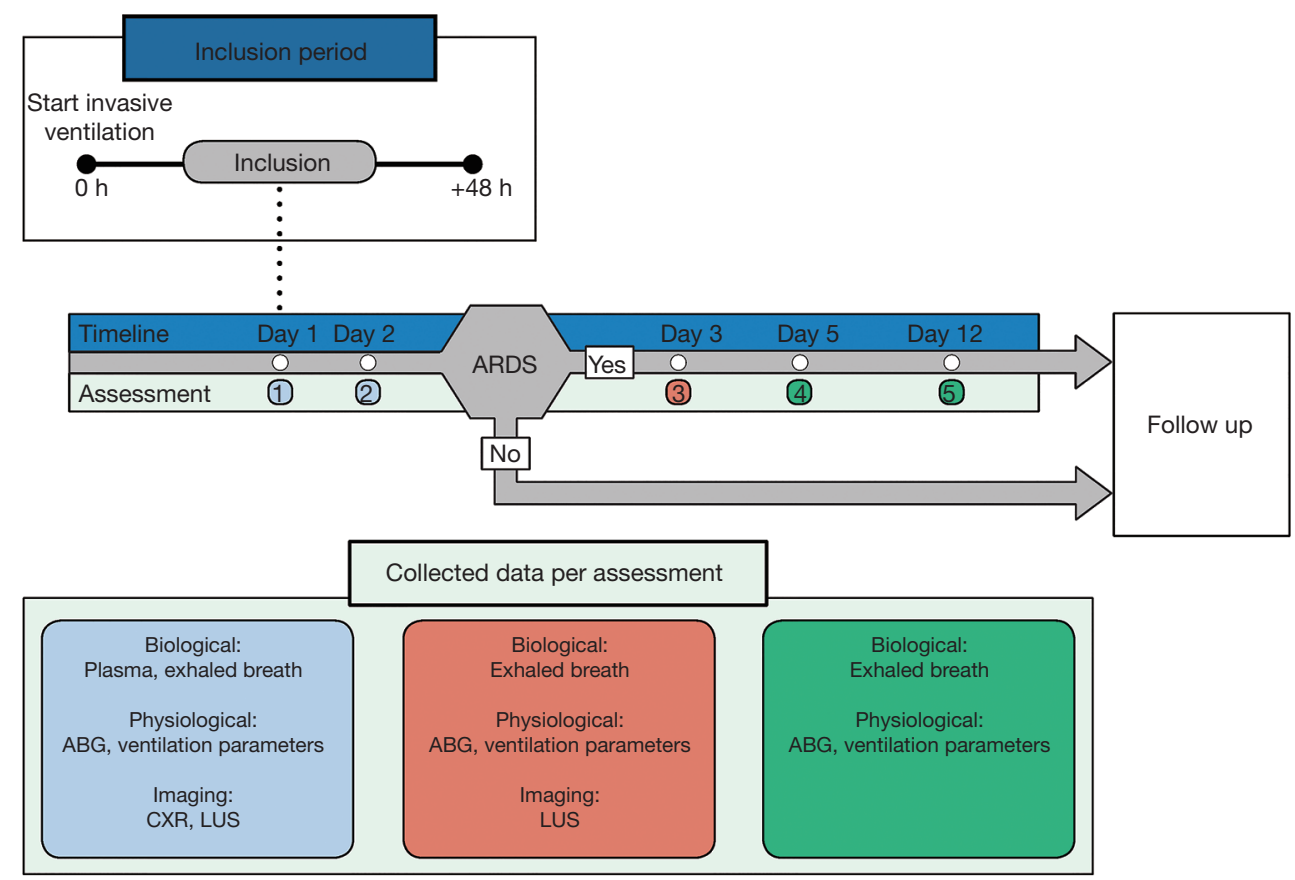

Figure 1 Overview of study procedures. Timeline with assessment 1 and 2 on day 1 and day 2. The researcher assesses if the patient fulfils the ARDS criteria at any time during the first 2 days. If the researcher diagnoses the patient with ARDS, the patient will undergo assessment 3 to 5 , as indicated with the arrow "yes", otherwise the patient will directly proceed to the follow up. In the box below the collected data per assessment is described. ARDS, acute respiratory distress syndrome; ABG, arterial blood gas; CXR, chest radiography; LUS, lung ultrasound.

fourth and fifth assessment consist of breath sampling and recording of clinical parameters only. Follow-up will end at the end of hospital stay and the following outcomes will be recorded: duration of ICU stay and hospital stay as well as ICU and hospital mortality.

\section{Classification of ARDS}

The diagnosis of ARDS will be based on the Berlin Definition and scored in three different settings (Figure 2). The first will regard ARDS as scored by the clinician: ARDS will be scored if ARDS is mentioned in the medical file. The second setting will be according to the researcher at inclusion and 1 day later, the researcher will use data of the 24 hours before assessment to score ARDS, this ARDS diagnosis is used to define if a day 3 assessment will be performed. The third setting will be assessment of ARDS by an expert panel in retrospect that will be blinded for the other clinical parameters. This panel will receive chest radiography, $\mathrm{PF}$ ratios, ventilator settings and ARDS risk factors. The chest radiography will be classified by a team of 3 experts, who will score and address the certainty of their decision (20). The ARDS scoring by the expert panel will be used as primary reference value for the assessment of diagnostic accuracy. In a subgroup analysis the diagnostic accuracy for the different categories of severity will be analysed. Risk factors for ARDS will be classified into the following categories: pneumonia, extrapulmonary sepsis, aspiration, trauma, inhalation, pancreatitis, pulmonary contusion, burn, pulmonary vasculitis, non-cardiogenic shock, drowning, drug overdose, blood transfusion, other risk factors (2). Additionally, ARDS will be divided into a hyper-inflammatory and hypo-inflammatory subphenotype based on a parsimonious classification score (21).

\section{Breath sampling}

Breath samples will be collected using a breath gas sampler (BGS) consisting of a pump (NMS020B 6VDC Micro Membranegas pump), a mass flow controller (Horiba STEC Z500), battery and charger (Panasonic LCRA1212PG and IDEAL POWER PC170-2) all combined 


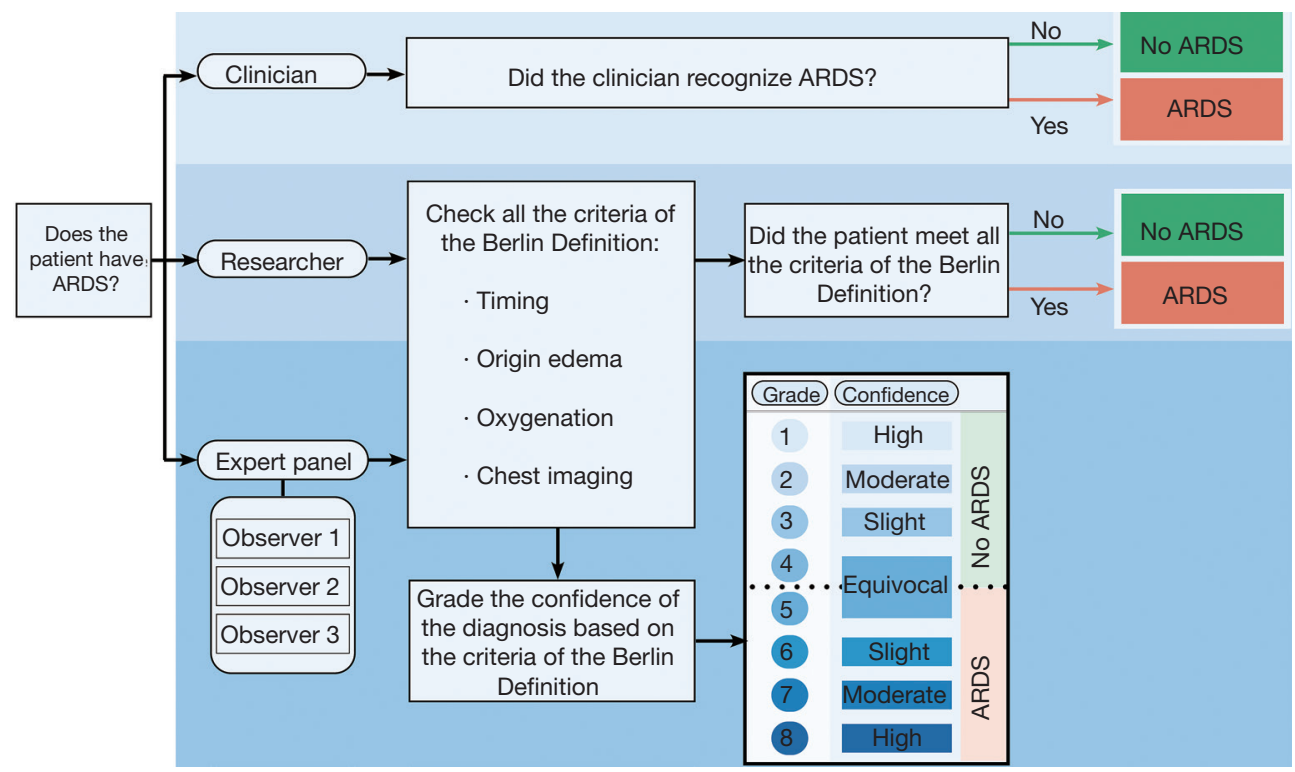

Figure 2 Classification of ARDS in three different settings; by the clinician, researcher and by an expert panel. The diagnosis of ARDS by the researcher is used to decide if additional measurements as shown in Figure 1 will be performed. The diagnosis of ARDS by the expert panel will be used as the reference value. ARDS, acute respiratory distress syndrome.

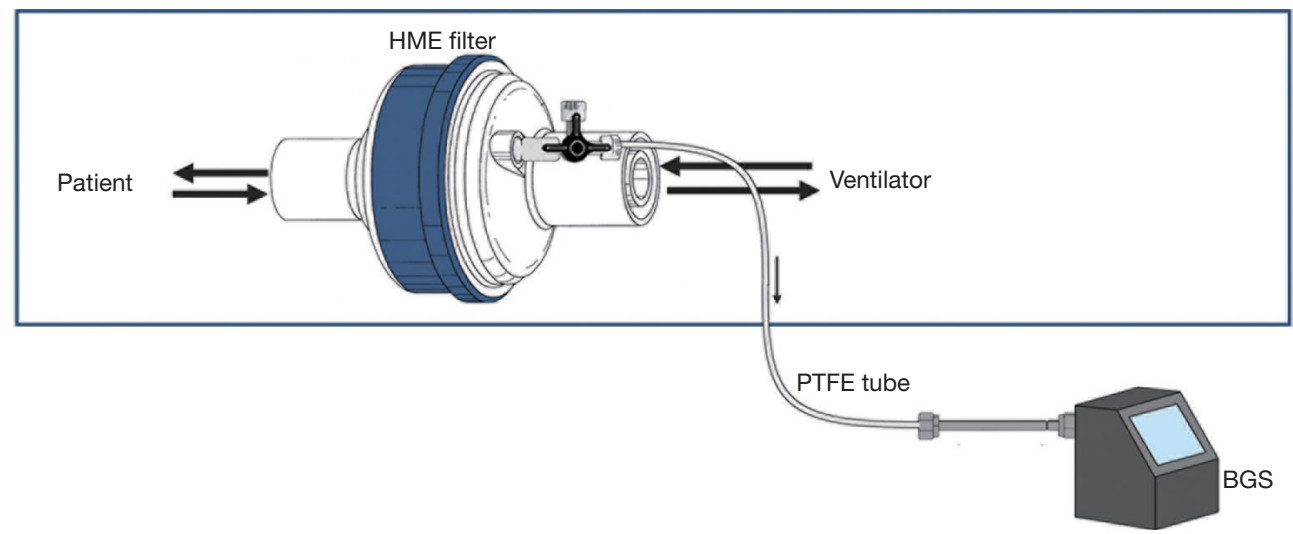

Figure 3 Breath sampling with a BGS through a side stream connection, distal from the HME filter. VOCs are collected on the sorbent tube. BGS, breath gas sampler; HME, heat-moisture exchange; VOCs, volatile organic compounds; PTFE, polytetrafluoroethylene.

in a metal casing with operating display (Brooks Instrument 0254). Exhaled breath will be drawn using this BGS and polytetrafluoroethylene (PTFE) tubing (Swagelok, Warrington, UK), by connecting a three way stop clock to the expiratory limb in case of double limb ventilation and after the heat-moisture exchange (HME) filter on the ventilator site in case of single limb ventilation. This method is adapted from Bos et al., reproducibility of this sampling method has been tested previously and showed a median intra-class correlation of 0.95 (22). Exhaled breath will be collected via this side-stream (Figure 3) during 6 minutes with a flow of $200 \mathrm{~mL} / \mathrm{min}$ (22). This is a completely non-invasive procedure, which has been performed several hundreds of times without any adverse events. VOCs in the exhaled breath will be absorbed onto two sorbent tubes filled with $300 \mathrm{mg}$ Carbograph 5TD (Markes International, Llantrisant, UK) and $90 \mathrm{mg}$ Tenax GR (Sigma-Aldrich Chemie B. V., Zwijndrecht, The 
Netherlands). Subsequently the sorbent tubes will be stored in the fridge at $4^{\circ} \mathrm{C}$.

\section{POC breath test}

A prototype POC breath test is specifically designed for the present study with the aim to quantify the amount of (n-) octane present in exhaled breath samples of ICU patients. The pathway from sample to result of the POC breath test will proceed as follows: the sorbent tube, containing the VOCs of the patient's exhaled breath will be inserted into a thermal desorption port. Inside this port, protected by thermal insulation, the sorbent tube will be heated to $200^{\circ} \mathrm{C}$ to transfer the VOCs to a thermal sorbent trap situated inside the POC device. Subsequently, this thermal trap will rapidly be heated to create an up-concentrated VOC mixture that will be split-injected into a GC column with dimethyl polysiloxane coating. The GC column will be used with the following temperature program: $40^{\circ} \mathrm{C}$-hold $30 \mathrm{~min}$, ramp $3{ }^{\circ} \mathrm{C} / \mathrm{min}$ for $10 \mathrm{~min}$ to $70^{\circ} \mathrm{C}$, hold $6.6 \mathrm{~min}$, ramp $20^{\circ} \mathrm{C} / \mathrm{min}$ to $170{ }^{\circ} \mathrm{C}$, hold 8.3 minutes and then cooling down with ramp $20^{\circ} \mathrm{C} / \mathrm{min}$ to $40^{\circ} \mathrm{C}$. This program gradually clears the column from all injected VOCs. As the VOCs exit the column, separated according to their volatility and varying interaction with the column coating, they will be recorded by a photo-ionization detector that ionizes the VOCs and quantifies the amount of resulting electrical charge. In particular the octane concentration will be quantified by considering the output of the detector at a specific time delay after injection and calibrated with respect to the photo-ionization efficacy for octane.

\section{GC-MS}

The other sorbent tube will be analysed by means of GCMS as described previously (23). In short, sorbent tubes will be heated to $280^{\circ} \mathrm{C}$ for $15 \mathrm{~min}$ with a flow of $30 \mathrm{~mL} / \mathrm{min}$. VOCs will be captured on a cold trap at $10^{\circ} \mathrm{C}$ and then reinjected by rapidly heating the trap to $300^{\circ} \mathrm{C}$ for 1 minute. Subsequently the molecules will be injected splitless through a transfer line at $180{ }^{\circ} \mathrm{C}$ onto an Inertcap $5 \mathrm{MS} / \mathrm{Sil}$ GC column [30 m, ID $0.25 \mathrm{~mm}$, film thickness $1 \mu \mathrm{m}$, 1,4-bis(dimethylsiloxy)phenylene dimethyl polysiloxane (Restek, Breda, The Netherlands)] with a flow of $1.2 \mathrm{~mL} / \mathrm{min}$. Oven temperature will be kept isothermal at $40{ }^{\circ} \mathrm{C}$ for 5 minutes, then increased to $280{ }^{\circ} \mathrm{C}$ at $10{ }^{\circ} \mathrm{C} / \mathrm{min}$ and kept isothermal at $280{ }^{\circ} \mathrm{C}$ for 5 minutes. Molecules will be ionized using electron ionization $(70 \mathrm{eV})$, and the fragment ions are detected using a quadrupole mass spectrometer (GCMS-GP2010, Shimadzu, Den Bosch, The Netherlands) with a scan range of 37-300 Da. GC-MS analysis, de-noising, peak detection, and alignment will be performed using the R "xcms" package (Scripps Center for Metabolomics, La Jolla, CA, USA) and will result in an ion fragment peak table as input for statistical analysis.

\section{Arterial blood gas analysis and LUS}

Arterial blood will be analysed through POC blood gas analysis. After this measurement, approximately $2 \mathrm{cc}$ of heparinized blood will be available as waste material. This blood will be centrifuged and the plasma will be stored at $-80{ }^{\circ} \mathrm{C}$. At the end of the study, the plasma will be thawed and pulmonary biological markers will be measured to determine the biological phenotype (15).

LUS will be performed with a linear transducer according to a previously published protocol (14). To ascertain good inter-rater reliability, all researchers will be trained by experienced physicians in LUS examination beforehand. Previous research has shown that this leads to an excellent inter-rater variability with an intraclass correlation coefficient (ICC) of 0.98 (24). Six examination regions will be selected per hemi thorax. Anterior axillary line and posterior axillary line divide the chest into anterior, lateral and posterior (25). The chest regions examined will contain point 1 and 2 on the anterior side, point 3 and 4 on the lateral side and point 5 and 6 on the posterior side (26). Each chest zone will be scanned, the most pathologic finding will be saved and scoring will be performed according to the regional aeration score (27).

\section{Study outcomes}

The primary endpoint of this study is the diagnostic accuracy of octane concentration in exhaled breath for ARDS defined by the panel of experts, depicted by the value which maximizes the specificity. The diagnostic accuracy will be described by the area under the receiver operating characteristics curve (AUROCC) and the optimal cut-off with sensitivity, specificity and likelihood ratios. Secondary endpoints are: (I) the association between exhaled breath octane and ARDS corrected for confounders and (II) the added diagnostic value of the breath test on top of the LIPS.

Tertiary endpoints are (I) dynamics of breath biomarkers over time and relation with outcome, (II) agreement of ARDS diagnosis between the researcher, clinician and 
an expert panel, (III) the diagnostic accuracy of LUS for ARDS, (IV) combined diagnostic accuracy of LUS and exhaled breath analysis for ARDS, (V) association between breath biomarkers and ARDS subphenotypes, (VI) association between breath biomarkers and plasma biomarkers in ARDS pathophysiology and (VII) association between the certainty of the ARDS diagnosis and the octane concentration.

\section{Sample size calculation}

With an expected sensitivity of approximately $80 \%$ (8) and a minimally acceptable lower confidence limit of $65 \%$ at least 52 patients with ARDS will be required for the study (28). At a predicted incidence of $10.4 \%$ (2) at least 500 patients will be needed for the primary endpoint.

\section{Statistical analysis}

Data will be summarised based on the type of data and the distribution of the values for continuous variables. Categorical variables will be presented as number and percentage and differences will be tested with a Chi square test. Continuous variables will be presented as mean with standard deviation for variables with a normal distribution (based on histograms, before and after transformation) or with a presumed normal distribution (such as height) and differences between groups will be tested with a $t$-test or one-way ANOVA, as appropriate. Continuous variables without a normal distribution (as defined above) will be presented as median, 25 th percentile and 75 th percentile and differences between groups will be tested with a MannWhitney U test or Kruskall Wallis test, as appropriate. Correlation between two continuous variables will be performed with Pearson correlation for normally distributed variables and with Spearman correlation for non-normally distributed variables.

Logistic regression will be performed with the $\mathrm{R}$ "rms" package. Univariate analysis will be performed for the primary and secondary outcomes. Multivariate analysis will always be performed with a selection of variables that have clinical merit to which statistically significant variables will be added that were not pre-defined. Automatic backward selection based on Akaike Information Criterion (AIC) will be performed for variable selection. Confounding is defined as a change in beta coefficient of $>10 \%$ after the addition of the potential confounder to the logistic regression model. Modification is defined as a statistically significant interaction term in the regression model. When modification is found, a stratified analysis will be performed within the subgroups.

Diagnostic accuracy is defined as the AUROCC. The optimal cut-off will be calculated via the Youden index and the corresponding sensitivity and specificity will be reported. Calibration will be assessed through a plot of observed probability against predicted probability with a smoothed LOESS line. The added value of combination of diagnostic tests over single tests will be assessed by netreclassification improvement and integrated discrimination index (29).

\section{End of study definition}

The DARTS study will end when the 2-year inclusion period is completed and at least 500 patients are included.

\section{Reporting}

The results will be reported according to the Standards for Reporting Diagnostic Accuracy (STARD) guidelines (30). The main manuscript will focus on the primary and secondary outcomes. Tertiary outcomes will be addressed in separate manuscripts.

\section{Discussion}

In this manuscript we described the protocol of the DARTS study, an observational multicentre study in mechanically ventilated ICU patients, aimed to assess diagnostic accuracy of octane in exhaled breath for ARDS. To our knowledge, this is the first study with an adequate sample size validating a biological marker that was discovered using untargeted analysis to non-invasively diagnose ARDS. The major novelty of this study is the use of a POC breath test that has been specifically developed for the purpose of diagnosing ARDS.

This study will have several strengths compared to previous studies on diagnostic tests for ARDS. First, we will assess patients in an early phase of invasive mechanical ventilation, as it is known that ARDS mostly develops during the first 2 days (31). Second, the recruited patient population includes all patients in two centers at risk for ARDS, which limits the likelihood of selection bias. Third, since the POC breath test might be used for monitoring disease progress in the future, longitudinal sampling is performed. Serial sampling will give insight in the changes 
in breath pattern during progression or resolution of lung injury. Fourth, to overcome the low reliability of ARDS diagnosis caused by the low interrater agreement, mostly caused by chest radiography interpretation, an expert panel is asked to diagnose ARDS independently. It is known that this increases the reliability of the diagnosis (20).

To assess the primary endpoint, diagnostic accuracy for ARDS, we require a quantitative assessment of the concentration of octane in exhaled breath of mechanically ventilated ICU patients. This concentration is derived from features of the POC breath test chromatogram. The procedure of analysis and data extraction is extensively tested and validated in a pilot setting and the results will be published before finalization of the here described study. Several important challenges will be addressed: stability of the column and detector over time, co-elution by VOCs with similar chemical properties and repeatability of breath sampling and analysis. For this purpose, calibration samples with varying concentrations of octane and several other VOCs scattered throughout the chromatogram will be analysed each week.

Next to answering the primary research questions, additional analysis will be performed to discover possible new biomarkers or features relevant in ARDS. This analysis will be conducted using plasma biomarkers, breath biomarkers and imaging data. Exhaled breath metabolomics data, analysed by GC-MS, will be used to evaluate if the same breath biomarkers that were previously found to discriminate patients with ARDS can be replicated in this cohort (8). Next to that, we will search for other VOCs that discriminate the breath of patients with ARDS using data obtained by GC-MS and the POC breath test. Subphenotyes will be discovered using unsupervised analysis of breath metabolomics and plasma biomarkers data. The LUS clips are systematically scored and are analysed for their diagnostic accuracy for ARDS and their association with outcome in conjunction with other imaging modalities. This will result in a comprehensive physiological, morphological and biological picture of each patient and refines the comparison of the breath test.

The heterogeneity in ARDS yields it unlikely that a single biomarker will provide perfect diagnostic results. However, octane in exhaled breath likely directly reflects oxidative stress in the lungs, which is one of the pathophysiological processes that can result in lung injury. It is known that changes in host response occur in acutely injured lung before they can be detectable in the systemic circulation (32). Therefore, a breath biomarker may provide us with early information about pathophysiological processes in the lungs, and can serve as an early warning sign of the development of lung injury in general but may also point into the direction of the etiological pathway that contributes to that injury. Besides diagnosis of ARDS, a biomarker may also have value when it identifies a subphenotype that consistently responds to a specific type of treatment, a so-called treatable trait (33).

In conclusion, we hypothesize that the octane concentration in exhaled breath, as measured with the POC breath test, can facilitate early diagnosis of ARDS in mechanically ventilated ICU patients.

\section{Acknowledgments}

Funding: The study is funded by Health Holland via the Dutch Lung Foundation. They had no role in the study design. And they will have no role in data collection, analysis and interpretation.

\section{Footnote}

Peer Review File: Available at https://dx.doi.org/10.21037/ atm-21-1384

Conflicts of Interest: All authors have completed the ICMJE uniform disclosure form (available at https://dx.doi. org/10.21037/atm-21-1384). ARMV, TMEN, and IG are employed by company Philips. RMS received grant for the research project, Lung fund, The Netherlands. LDJB received grants from Health Holland, Dutch Lung Foundation (longfonds), Innovative medicine initiative and Amsterdam UMC. The other authors have no conflicts of interest to declare.

Ethical Statement: The authors are accountable for all aspects of the work in ensuring that questions related to the accuracy or integrity of any part of the work are appropriately investigated and resolved. The Institutional Review Board of the Academic Medical Center, Amsterdam, The Netherlands, and of the Maastricht University Medical Center+, Maastricht, The Netherlands, waived the need for ethical approval of the protocol (W18_311 \#18.358 and 2019-1137). The biobank review board of the Academic Medical Center Biobank approved the development of a biobank for the study (approval 2018_287\#A201921). Patients will be recruited as soon as possible after start of mechanical ventilation by a team of researchers. Therefore, 
all included patients are, per definition, incapacitated to provide informed consent. All measurements in this study are non-invasive and data needs to be collected shortly after intubation. For this reason, informed consent cannot be obtained from the patient beforehand and deferred consent is asked as the alternative. Patients are informed and asked for written consent shortly after regaining the capacity to provide consent. If the patient declines participation in the study, the collected data will be destroyed. If the patient deceases, the General Data Protection Regulation (GDPR) states that the obtained data can be used without consent. If a patient does not regain capacity before discharge, the representative is asked for deferred consent. In case the representative does not give consent, the collected data will be destroyed. When the representative could not be reached, and the patient does not regain capacity, the patient will be excluded and the collected data will be destroyed. In case the representative gave consent, the patient has the possibility for an opt-out procedure. In all cases data will be destroyed if consent is not given by either the patient or the representative.

Open Access Statement: This is an Open Access article distributed in accordance with the Creative Commons Attribution-NonCommercial-NoDerivs 4.0 International License (CC BY-NC-ND 4.0), which permits the noncommercial replication and distribution of the article with the strict proviso that no changes or edits are made and the original work is properly cited (including links to both the formal publication through the relevant DOI and the license). See: https://creativecommons.org/licenses/by-nc-nd/4.0/.

\section{References}

1. Sweeney RM, McAuley DF. Acute respiratory distress syndrome. Lancet 2016;388:2416-30.

2. Bellani G, Laffey JG, Pham T, et al. Epidemiology, patterns of care, and mortality for patients with acute respiratory distress syndrome in intensive care units in 50 countries. JAMA 2016;315:788-800.

3. ARDS Definition Task Force; Ranieri VM, Rubenfeld GD, et al. Acute respiratory distress syndrome: the Berlin Definition. JAMA 2012;307:2526-33.

4. Goddard SL, Rubenfeld GD, Manoharan V, et al. The randomized educational acute respiratory distress syndrome diagnosis study: a trial to improve the radiographic diagnosis of acute respiratory distress syndrome. Crit Care Med 2018;46:743-8.
5. Bellani G, Pham T, Laffey JG. Missed or delayed diagnosis of ARDS: a common and serious problem. Intensive Care Med 2020;46:1180-3.

6. Binnie A, Tsang JL, dos Santos CC. Biomarkers in acute respiratory distress syndrome. Curr Opin Crit Care 2014;20:47-55.

7. Moser B, Bodrogi F, Eibl G, et al. Mass spectrometric profile of exhaled breath--field study by PTR-MS. Respir Physiol Neurobiol 2005;145:295-300.

8. Bos LD, Weda H, Wang Y, et al. Exhaled breath metabolomics as a noninvasive diagnostic tool for acute respiratory distress syndrome. Eur Respir J 2014;44:188-97.

9. Kneepkens CM, Lepage G, Roy CC. The potential of the hydrocarbon breath test as a measure of lipid peroxidation. Free Radic Biol Med 1994;17:127-60.

10. Quinlan GJ, Lamb NJ, Evans TW, et al. Plasma fatty acid changes and increased lipid peroxidation in patients with adult respiratory distress syndrome. Crit Care Med 1996;24:241-6.

11. Carpenter CT, Price PV, Christman BW. Exhaled breath condensate isoprostanes are elevated in patients with acute lung injury or ARDS. Chest 1998;114:1653-9.

12. Hagens LA, Verschueren ARM, Lammers A, et al. Development and validation of a point-of-care breath test for octane detection. Analyst 2021;146:4605-14.

13. Trillo-Alvarez C, Cartin-Ceba R, Kor DJ, et al. Acute lung injury prediction score: derivation and validation in a population-based sample. Eur Respir J 2011;37:604-9.

14. Vercesi V, Pisani L, van Tongeren PSI, et al. External confirmation and exploration of the Kigali modification for diagnosing moderate or severe ARDS. Intensive Care Med 2018;44:523-4.

15. Calfee CS, Janz DR, Bernard GR, et al. Distinct molecular phenotypes of direct vs indirect ARDS in single-center and multicenter studies. Chest 2015;147:1539-48.

16. Calfee CS, Delucchi K, Parsons PE, et al. Subphenotypes in acute respiratory distress syndrome: latent class analysis of data from two randomised controlled trials. Lancet Respir Med 2014;2:611-20.

17. Bos LD, Schouten LR, van Vught LA, et al. Identification and validation of distinct biological phenotypes in patients with acute respiratory distress syndrome by cluster analysis. Thorax 2017;72:876-83.

18. Knaus WA, Draper EA, Wagner DP, et al. APACHE II: a severity of disease classification system. Crit Care Med 1985;13:818-29.

19. Vincent JL, Moreno R, Takala J, et al. The SOFA (Sepsis- 
related Organ Failure Assessment) score to describe organ dysfunction/failure. On behalf of the Working Group on Sepsis-Related Problems of the European Society of Intensive Care Medicine. Intensive Care Med 1996;22:707-10.

20. Sjoding MW, Hofer TP, Co I, et al. Interobserver reliability of the Berlin ARDS definition and strategies to improve the reliability of ARDS diagnosis. Chest 2018;153:361-7.

21. Sinha P, Delucchi KL, McAuley DF, et al. Development and validation of parsimonious algorithms to classify acute respiratory distress syndrome phenotypes: a secondary analysis of randomised controlled trials. Lancet Respir Med 2020;8:247-57.

22. Bos LD, Wang Y, Weda H, et al. A simple breath sampling method in intubated and mechanically ventilated critically ill patients. Respir Physiol Neurobiol 2014;191:67-74.

23. Bos LD, van Walree IC, Kolk AH, et al. Alterations in exhaled breath metabolite-mixtures in two rat models of lipopolysaccharide-induced lung injury. J Appl Physiol (1985) 2013;115:1487-95.

24. Smit MR, de Vos J, Pisani L, et al. Comparison of linear and sector array probe for handheld lung ultrasound in invasively ventilated ICU patients. Ultrasound Med Biol 2020;46:3249-56.

25. Via G, Storti E, Gulati G, et al. Lung ultrasound in the ICU: from diagnostic instrument to respiratory monitoring tool. Minerva Anestesiol 2012;78:1282-96.

Cite this article as: Hagens LA, Heijnen NFL, Smit MR, Verschueren ARM, Nijsen TME, Geven I, Schultz MJ, Bergmans DCJJ, Schnabel RM, Bos LDJ; on behalf of the DARTS consortium. Diagnosis of acute respiratory distress syndrome (DARTS) by bedside exhaled breath octane measurements in invasively ventilated patients: protocol of a multicentre observational cohort study. Ann Transl Med 2021;9(15):1262. doi: 10.21037/atm-21-1384
26. Kruisselbrink R, Chan V, Cibinel GA, et al. I-AIM (Indication, Acquisition, Interpretation, Medical Decisionmaking) framework for point of care lung ultrasound. Anesthesiology 2017;127:568-82.

27. Volpicelli G, Elbarbary M, Blaivas M, et al. International evidence-based recommendations for point-of-care lung ultrasound. Intensive Care Med 2012;38:577-91.

28. Flahault A, Cadilhac M, Thomas G. Sample size calculation should be performed for design accuracy in diagnostic test studies. J Clin Epidemiol 2005;58:859-62.

29. Pencina MJ, D'Agostino RB Sr, Steyerberg EW. Extensions of net reclassification improvement calculations to measure usefulness of new biomarkers. Stat Med 2011;30:11-21.

30. Bossuyt PM, Reitsma JB, Bruns DE, et al. Towards complete and accurate reporting of studies of diagnostic accuracy: the STARD initiative. BMJ 2003;326:41-4.

31. Laffey JG, Bellani G, Pham T, et al. Potentially modifiable factors contributing to outcome from acute respiratory distress syndrome: the LUNG SAFE study. Intensive Care Med 2016;42:1865-76.

32. Pugin J, Verghese G, Widmer MC, et al. The alveolar space is the site of intense inflammatory and profibrotic reactions in the early phase of acute respiratory distress syndrome. Crit Care Med 1999;27:304-12.

33. Agusti A, Bel E, Thomas M, et al. Treatable traits: toward precision medicine of chronic airway diseases. Eur Respir J 2016;47:410-9. 